Supporting Information for:

\title{
Tryptophan 232 within Oxidosqualene-Lanosterol Cyclase from Saccharomyces cerevisiae Influences Rearrangement and Deprotonation but not Cyclization Reactions \\ Tung-Kung $\mathrm{Wu}^{* \mathrm{a}}$, Mei-Ting Yu ${ }^{\dagger a}$, Yuan-Ting Liu ${ }^{\dagger a}$, Cheng-Hsian Chang ${ }^{\dagger a}$, Hsing-Ju Wang $^{\mathrm{a}}$, and Eric Wei-Guang Diau ${ }^{\mathrm{b}}$
}

${ }^{a}$ Department of Biological Science and Technology

${ }^{b}$ Department of Applied Chemistry

National Chiao Tung University

300, Hsin-Chu, Taiwan (Republic of China)

\section{Materials and methods}

Constructing Site-Saturated Mutagenic Plasmids. The plasmid pTKERG7RS314WT was used as a template to construct the $E R G 7^{\text {W232X }}$ site-saturated mutagenesis plasmids. The mutagenic oligonucleotide primers were MTYOSCW232X1 (5'-CCCATGCATCCCGGGAGATGGNNNGTTCATACT-3'; the Sma I site is underlined) and MTYOSCW232X2 (5'-AGTATGAACNNNCCATCTCCCGGGATG CATGGG-3'; the Sma I site is underlined). Site-saturated mutagenic DNAs were constructed by use of PCR amplification with the QuickChange Site-Directed Mutagenesis kit (Stratagene Inc., La Jolla, CA), according to the manufacturer's instructions. Briefly, PCR was performed with MTYOSCW232X1 and MTYOSCW232X2 primers using Pfu DNA polymerase with $10 \mathrm{mM}$ of each of the following: dNTP, $1 \mu$ g each primer, $1.5 \mu \mathrm{L}$ glycerol, $2 \mu \mathrm{L}$ pTKERG7RS314WT template, $5 \mu \mathrm{L} 10$ X Pfu reaction buffer, and $\mathrm{ddH}_{2} \mathrm{O}$ to create a final volume of $50 \mu \mathrm{L}$. The reaction mixture was denaturated at $95^{\circ} \mathrm{C}$ for $2 \mathrm{~min}$, and then run for 18 cycles of denaturing at $95^{\circ} \mathrm{C}$ for $30 \mathrm{sec}$ each, annealing at $56^{\circ} \mathrm{C}$ for $1 \mathrm{~min}$, with an extension at $68^{\circ} \mathrm{C}$ for $16 \mathrm{~min}$. Then $1 \mu \mathrm{L}$ of $\mathrm{Dpn}$ I restriction enzyme was added to the mixture 
and the mixture was incubated at $37^{\circ} \mathrm{C}$ for $4 \mathrm{~h}$ to digest the parental supercoiled DNA. The mutated plasmids were used to transform competent Epicurian coli ${ }^{\circledR}$ XL1-Blue cells (Strategene, La Jolla, CA). Plasmid DNAs were purified from the batch cultures by use of a DNA mini-prep kit, and were then digested with Sma I, to confirm the presence of the mutations. The introduction of a Sma I silent mutation cleaved the once circular plasmid into a linear DNA fragment of $7.23 \mathrm{kbp}$. The exact amino acid substitution at W232 position was determined by sequence of the DNA by use of an ABI PRISM 3100 auto-sequencer. Samples were prepared with the appropriate oligonucleotides as primers and an ABI Prism BigDye Terminator Cycle Sequencing Ready Reaction Kit, according to the manufacturer's protocol (Applied Biosystems, Foster City, CA, USA).

Transformation of $p E R G 7^{\text {W232X }}$ Plasmids into Yeast Strain TKW14. The pERG7 $^{\mathrm{W} 232 \mathrm{X}}$ mutated plasmids were transformed into TKW14 by electroporation using a GenPulser with Pulse Controller (BioRad, Hercules, CA). The pRS314 and pTKERG7RSWT plasmids were also transformed as negative and positive controls, respectively. The $\begin{array}{llll}\text { resulting transformants } & \text { were }\end{array}$ SD+Ade+Lys+His+Met+Ura+hemin+G418+Erg plates, and incubated for three to five days to select for the presence of pRS314-derived plasmids. The transformants were

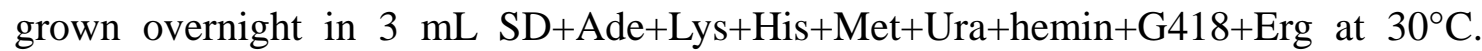
Aliquots of $100 \mu \mathrm{L}$ of each culture were plated on SD+Ade+Lys+Trp+His+Met+Ura+hemin+G418 and incubated at $30^{\circ} \mathrm{C}$ for three to five days to confirm the complementation effect.

Extracting Lipids and Characterizing Mutant Products. TKW14[pERG7 $\left.{ }^{\mathrm{W} 232 \mathrm{X}}\right]$ transformants
Two liter cultures of

were grown 
SD+Ade+Lys+His+Ura+Met+Hemin+Erg medium at $30^{\circ} \mathrm{C}$ with shaking (220 rpm) for five days. The cells were harvested by centrifugation, washed, and saponified by refluxing them in $1 \mathrm{~L}$ of a $15 \% \mathrm{KOH} / 50 \% \mathrm{EtOH}$ for $3 \mathrm{~h}$. Two volumes of water were added and the nonsaponifiable lipid (NSL) was thrice extracted with petroleum ether $\left(40-60^{\circ} \mathrm{C}\right)$. The petrol extract was washed with water, dried over with anhydrous sodium sulfate, filtered and dried thoroughly in a rotary evaporator. The extract was fractionated by silica gel column chromatography using a 19:1 hexane/ethyl acetate mixture.

GC and GC/MS Conditions. GC analyses were performed with a Hewlett-Packard model 5890 series II or Agilent 6890N chromatograph equipped with a DB-5 column (30 m x $0.25 \mathrm{~mm}$ I.D., $0.25 \mu \mathrm{m}$ film; oven gradient at $50^{\circ} \mathrm{C}$ for $2 \mathrm{~min}$, and then $20^{\circ} \mathrm{C}$ per min until $300^{\circ} \mathrm{C}$, held at $300^{\circ} \mathrm{C}$ for $20 \mathrm{~min}, 300^{\circ} \mathrm{C}$ injector; $250^{\circ} \mathrm{C}$ interface; $1 / 40$ split ratio using helium carrier gas at 13 psi column head pressure). GC/MS was performed on a Hewlett-Packard model 5890 II GC (J \& W DB-5MS column, 30 m x $0.25 \mathrm{~mm}$ I.D., $0.25 \mu \mathrm{m}$ film; oven $280^{\circ} \mathrm{C}$, injector $270^{\circ} \mathrm{C}$, GC-MS transfer line: $280^{\circ} \mathrm{C}$ ) coupled to a TRIO-2000 micromass spectrometer $\left(\mathrm{EI}^{+}, 70 \mathrm{eV}, 180^{\circ} \mathrm{C}\right.$ ion source temperature, 30-650 amu scan range).

Lanosteryl acetate. The GC relative retention time for lanosterol (measured relative to ergosterol) was 1.64. MS for lanosteryl acetate, $m / z=468\left([\mathrm{M}]^{+}\right), 453,393,339,301$, 281, 255, 241, 229, 189, 159, 109, 95, 81, 69 (100), $55 . \quad$ The GC and MS data were consistent with those published in the literature. ${ }^{13} \mathrm{C}$ NMR $(150.68 \mathrm{MHz}, 30 \mathrm{mM}$ solution in $\left.\mathrm{CDCl}_{3}, 25^{\circ} \mathrm{C}\right) \delta 15.74$ (C-18), 16.51 (C-29), 17.62 (C-27), 18.11 (C-6),

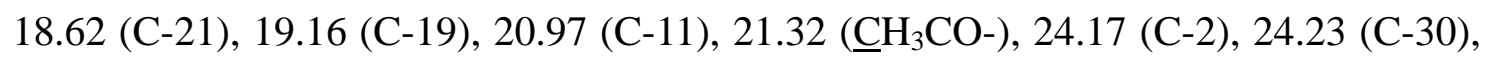
24.92 (C-23), 25.74 (C-26), 26.37 (C-7), 27.90 (C-28), 28.18 (C-16), 30.81 (C-15), 30.96 (C-12), 35.26 (C-1), 36.25 (C-20), 36.34 (C-22), 36.89 (C-10), 37.80 (C-4), 44.47 (C-13), 49.80 (C-14), 50.38 (C-17), 50.50 (C-5), 80.96 (C-3), 125.24 (C-24), 
130.87 (C-25), 134.24 (C-9), 134.49 (C-8), 171.02 ( $\left.\mathrm{CH}_{3} \underline{\mathrm{C} O}-\right)$. Chemical shifts $( \pm 0.02$ $\mathrm{ppm}$ ) were referenced to the $\mathrm{CDCl}_{3}$ signal at $\delta 77.0 \mathrm{ppm}$.

Parkeyl acetate. The GC relative retention time for parkeol (measured relative to ergosterol) was 2.34. MS for parkeyl acetate, $m / z=468\left([\mathrm{M}]^{+}\right), 453,393,407,355$, 295, 207, 173, 159, 133, 109, 95, 81, 69 (100), $55 .{ }^{13} \mathrm{C}$ NMR (150.68 MHz, $30 \mathrm{mM}$ solution in $\left.\mathrm{CDCl}_{3}, 25{ }^{\circ} \mathrm{C}\right) \delta 14.39$ (C-18), 16.78 (C-29), 17.62 (C-27), 18.28 (C-21), 18.47 (C-30), 21.23 (C-6), 21.32 ( CH$\left._{3} \mathrm{CO}-\right), 22.28$ (C-19), 24.15 (C-2), 24.92 (C-23), 25.70 (C-26), 28.00 (2C, C-7 \&C-16), 28.18 (C-28), 33.90 (C-15), 35.79 (C-1), 35.91 (C-20), 36.38 (C-22), 37.13 (C-12), 37.99 (C-4), 39.23 (C-10), 41.74 (C-8), 44.28 (C-13), 47.00 (C-14), 50.91 (C-17), 52.56 (C-5), 80.88 (C-3), 115.19 (C-11), 125.21 (C-24), 130.91 (C-25), 148.11 (C-9), 170.99 ( $\mathrm{CH}_{3}$ COO-). Chemical shifts $( \pm 0.02$ ppm) were referenced to the $\mathrm{CDCl}_{3}$ signal at $77.0 \mathrm{ppm}$. The NMR data were consistent with those published in the literature.

Protosta-12,24-dien-3 $\beta$-ol. ${ }^{1} \mathrm{H}$ NMR (600 MHz, $\left.\mathrm{CDCl}_{3}\right): \delta 5.276(\mathrm{t}, 1 \mathrm{H}, J=1.8 \mathrm{~Hz}$, H-12), 5.075 (t, 1H, $J=7$ Hz, H-24), 3.196 (dd, 1H, $J=11.5,4.7$ Hz, H-3 $\alpha$ ), 2.170 (br q, 1H, $J=7.8 \mathrm{~Hz}, 7.8 \mathrm{~Hz}, \mathrm{H}-17 \alpha$ ), 2.029 (m, 1H, H-23S), 1.909-1.1938 (m, 1H), 1.829-1.850 (m, 4H), 1.706-1.790 (m, 3H), 1.662 (s, 3H, Me-26), 1.644 (m, 2H, H-2), 1.588 (s, 3H, Me-27), 1.554-1.620 (m, 3H), 1.489-1.527 (m, 3H), 1.398-1.479 (m, 5H), 1.168-1.232 (m, 2H), 1.013 (s, 3H, Me-19), 0.954 (s, 3H, Me-18), 0.943 (s, 3H, Me-29), 0.905 (d, 3H, $J=6.76 \mathrm{~Hz}, \mathrm{Me}-21$ ), 0.878 (s, 3H, Me-30), 0.833 (s, 3H, Me-28). Chemical shifts were referenced to $\mathrm{Si}\left(\mathrm{CH}_{3}\right)_{4}$ and are generally accurate to + 0.001 ppm. ${ }^{13} \mathrm{C}$ NMR (150.77 MHz, $30 \mathrm{mM}$ solution in $\left.\mathrm{CDCl}_{3}, 25{ }^{\circ} \mathrm{C}\right) \delta 15.82$ (C-28), 17.68 (C-27), 18.17 (C-6), 18.27 (C-21), 19.92 (C-30), 22.65 (C-18), 25.67 (C-11), 25.72 (C-26), 25.89 (C-23), 26.29 (C-19), 26.99 (C-16), 28.44 (C-2), 28.48 (C-29), 30.21 (C-7), 31.59 (C-15), 34.11 (C-22), 35.04 (C-1), 36.08 (C-10), 37.56 (C-20), 37.59 (C-14), 39.61 (C-4), 45.19 (C-5), 45.42 (C-9), 49.43 (C-17), 51.95 
(C-8), 79.33 (C-3), 119.78 (C-12), 125.15 (C-24), 130.98 (C-25), 148.76 (C-13).

MS $m / z=426\left([\mathrm{M}]^{+}\right), 410,393,357,341,313,295,274,257,229,204,175,147,129$, 107, 86, 69 (100), 52. The GC relative retention time (measured relative to lanosterol) was -0.21 .

Atomic coordinates for quantum mechanical model of protosta-12,24-dien-3b-ol, lanosterol, parkeol, and proteryl C-13 cation.

Coordinates for geometries optimization were constructed and optimized at the B3LYP/6-31G* level using Gaussian 03 software. Coordinates are shown in condensed format and can be converted to tabular form using the popular used word processors. Paragraphs were replaced with “ | ” marks.

\section{$\mathrm{C}_{30} \mathrm{H}_{50} \mathrm{O}_{1}$ model for parkeol}

1 | 1 | GINC-RN15 | FOpt | RB3LYP | 6-31G(d) | C30H5001 | LINHAT | 14-Feb-2006 | 0 | | \# B3LYP/6-31G* FOPT GEOM=CHECK GUESS=READ IOP(1/8=5) | | parkeol $|\quad| \quad 0,1 \quad$ |

C, $-5.7954624294,-0.5359524702,0.1852441943 \mid C,-6.0615232194,-1.0165855336,-1.2347999644$

C, $-5.0820277401,-2.1377972554,-1.6983894974$ | C, $-3.6110322919,-1.6390494082,-1.4436388265$

C, $-3.2794854002,-1.0424640529,-0.0229077111 \mid C,-4.3678578712,-0.0009725144,0.3325871681$

C, $-2.5269467798,-2.6441900635,-1.8731191468$ | C, $-1.172240787,-1.9422655641,-1.988594763$

C, $-0.7401149399,-1.2565142285,-0.6746173469 \mid \mathrm{C},-1.8676221578,-0.4059448362,-0.0720484252$

C, $0.5712386912,-0.4366482239,-0.8168537128 \quad$ ～C, $0.8830404495,0.3206403266,0.5300185836$

C, $-0.2219052907,1.3769773374,0.7142732773 \quad$ ～C, $-1.598779885,0.7771065642,0.5077942163$

C, $1.8562585673,-1.2687053518,-1.0235266166$ | C,3.00483654,-0.3673653378,-0.4922029536

C, $2.3506479774,0.8206074905,0.2913085107 \mid 0,-7.433788354,-1.4141258871,-1.2824708471$

C, $-5.4291148843,-3.4846889306,-1.0307018835 \mid$ C $,-5.281565079,-2.3188528301,-3.2237276053$

C, $-3.2259938286,-2.0966575002,1.1195767185$ | C, $0.4429070582,0.5514530194,-2.0086214184$

C, $0.8600835196,-0.6318039144,1.7506929265 \quad$ C, $3.2091564865,1.285508275,1.4977762268$

C,4.619536225,1.700515735,1.0117056517｜， $, 5.6434240765,1.9870307583,2.1318511819$

C, $7.0364156079,2.1721407807,1.5900503086$ | C, $7.8189320676,3.2617983917,1.6320675575$

C,9.1965366119,3.2468426291,1.0114325016 | C, 7.4420836251,4.5745077448,2.2754997466

C, 2.5569926947,2.4322936496,2.2884541251 | H, -5.9931983983,-1.3509115189,0.8912023992

$\mathrm{H},-6.5173516951,0.2543878189,0.4221058046 \quad \mathrm{H},-5.908296736,-0.155699978,-1.9107816267$

$\mathrm{H},-3.5017372578,-0.7743117465,-2.1201638785 \quad$ | H, $-4.224372023,0.3568616646,1.3591309233$

H, $-4.2498402137,0.8740637352,-0.321590707 \quad \mid \quad H,-2.7749741633,-3.0881548126,-2.8431129167$

H, $-2.4655988546,-3.4784996094,-1.1635600999 \mid \mathrm{H},-1.2514084887,-1.2010221886,-2.7918998872$

H, $-0.3961449042,-2.6559546707,-2.2949384581 \quad$ | H, $-0.5354541945,-2.077304906,0.0309795576$ 
H, $-0.0668104305,2.2265923484,0.0320966145$

H, - 2.4103847666, 1.3598470156,0.9347714914

H, $1.8051073972,-2.204164111,-0.453947127$

H, 3.6680567527, - - 0.940184995,0.1670992265

H, - 7.6737373621, - 1.5535452006, -2.2107711805

H, $-6.4280565153,-3.8056820679,-1.3403059595$

H, - 4.6963816455, - 3.1525673636, - 3.6230597423

H, - 6.3301210322, - 2.5458483464, - 3.4559277277

H, $-2.5261007444,-2.9109425195,0.9137340516$

H, 1.2626432527, 1.2732507521, - 2.0419129813

H, $0.4739897139,0.0048393164,-2.9561038361$

H, $1.5594189764,-1.4674786203,1.6457201735$

H, 3.3411556688,0.4307062663,2.1784505195

H, $5.0325463404,0.9074232752,0.377288677$

H, 5.3353196618, 2.857634366, 2.717839634

H, $9.9714332545,3.4677633688,1.7593921036$

H, $9.4302837104,2.278145405,0.5583422595$

H, 8.1516708835, 4.8335441245, 3.0739895068

H, 3.2052021536,2.7762759594,3.1009479064

H, 2.3578257124,3.2929190594,1.6364201887

$\mathrm{HF}=-1248.5009564|\mathrm{RMSD}=6.832 \mathrm{e}-09| \mathrm{RMSF}=3.013 \mathrm{e}-06 \mid$ Dipole $=0.3682176,0.0508477,-0.435191$ $\mathrm{PG}=\mathrm{C} 01[\mathrm{X}(\mathrm{C} 30 \mathrm{H} 5001)]$ | | @

\section{$\mathrm{C}_{30} \mathrm{H}_{50} \mathrm{O}_{1}$ model for lanosterol}

1 | 1 | GINC-RN15 | FOpt | RB3LYP | 6-31G(d) | C30H5001 | LINHAT | 16-Feb-2006 | 0 | | \# B3LYP/6-31G* FOPT

GEOM=CHECK $\mid$ | lanos terol |

766 | C, -4.8962636611, - 2.0791072175, -2.1854724262 | C, - 3.4618364149, - 1.611274298, - 1.7496186975 | C, -3.3321005357, -0.8223371329,-0.3976464047 | C, - 4.3644667836,0.3378265777, -0.4292454199 | C $,-2.386766119,-2.7053361968,-1.8107438316|\mathrm{C},-0.9874549634,-2.0780916308,-1.8088566493| \mathrm{C},-0$ $.8441587241,-0.8666445031,-0.9134725763|\mathrm{C},-1.8867953146,-0.2705745266,-0.2984602729| \mathrm{C}, 0.54$ 75070368,-0.2435504335,-0.8253020708 | C, $0.7978702302,0.3848087859,0.5975267124 \mid \mathrm{C},-0.2539037$ $182,1.483625325,0.7956446933|\mathrm{C},-1.6960827718,0.9375374617,0.6156026847| \mathrm{C}, 1.7766534369,-1.1$ $706594729,-0.9705628406$ | C, $2.9380544322,-0.3591697609,-0.3315186919$ | C, 2.3011957361,0.810879 4902,0.4974035374| 0,-7.217260447,-1.1713277132,-2.3314326754|C, -5.4603191336,-3.2670198992 $,-1.3795632868|\mathrm{C},-4.8422063462,-2.5185376744,-3.6694907584| \mathrm{C},-3.5796546973,-1.6721789885,0$ $.8792396042|\mathrm{C}, 0.6281278392,0.7934536339,-1.9862202392| \mathrm{C}, 0.6382230088,-0.6754423041,1.71619$ 12035 | C, 3.0971330757,1.1187525282,1.7939659735 | C,4.5575472182,1.4961511852,1.4427805255 |C 
,5.52836967,1.5328714619,2.6431109427| C,6.958089048,1.7258967983,2.2109058034 | C, 7.79805530 23,2.7321266523,2.4996251115 | C,9.2065994694,2.742404771,1.9527010553 | C, 7.4608350169,3.9202 357448,3.3678002992 | C, 2.4546161956,2.2231009804,2.650232328| H, -6.1839512656, -0.7844714169, $0.0215038827 \mid$ H, -6.4620029668,0.7362394005,-0.8027255002| H, -5.5074002688, -0.12930602,-2.875 $7234026 \mid$ H, -3.1797133438,-0.8665145365,-2.5127014801| H, -4.3760851052,0.8642825104,0.5317201 $581|\mathrm{H},-4.0499657933,1.0737554274,-1.1835645685| \mathrm{H},-2.4956984912,-3.3234485889,-2.7075533889$ | H, -2.4908934035,-3.3873618393,-0.9595092446| H, - 0.7204204923,-1.7872749763,-2.8381653125 | H, - - .2442744449, - 2.8360353888, - 1.5277651734| H, - 0.0714670631 ,2.2961171057,0.0836708429| H, -0 .1823960438,1.9367189787,1.7891792828| H, -2.0989527581,0.6772299092,1.6054308581| H, -2.32990 13259,1.763291936,0.2699320102| H, 1.9819596148,-1.4332177918,-2.0143031949| H, 1.6259517188, 2.1128431677,-0.4334870521|H,3.6172518389,0.0287150315,-1.0975498971| H,3.5445259897,-1.004 0854158,0.3152835157| H,2.3401267541,1.7289455174, -0.1056013921 | H, - 7.3061495642, -1.46766540 $99,-3.249660274|\mathrm{H},-4.7745705529,-4.1207965998,-1.4108428361|$ H, -6.4152144417, -3.5864581989, $-1.8074645461|\mathrm{H},-5.6469286088,-3.0279717146,-0.3313862184| \mathrm{H},-4.2702943408,-3.4410288679,-3$ $.8071038547|\mathrm{H},-4.3934408353,-1.7450796936,-4.3054518074| \mathrm{H},-5.8497065476,-2.7266952398,-4.0$ $524738857|\mathrm{H},-3.2865722945,-1.102310517,1.7679366027| \mathrm{H},-2.9914889307,-2.5938546779,0.879610$ $0081 \mid \mathrm{H},-4.6293038515,-1.9438801904,1.0123457492$ | H, 1.605496973,1.2822252172,-2.0550176322 | H, - 0.138429071 , 1.5683748752, - 1.90767636| H,0.4633855745,0.2747233578, - 2.9379284009| H,0.7064 007999,-0.2007761626,2.7013396977| H,1.4147111576, - 1.4456899976,1.6771832221 | H, - 0.327984965 1,-1.1819865551,1.6586351221| H,3.1363242615,0.1989108218,2.3970312421 | H,4.5693049268,2.475 0186577,0.9409974332| H,4.9558823496,0.7796784318,0.7146683449| H, 5.4458135922,0.5749077121, 3.1812267099| H, 5.2285984917,2.3077150872,3.3546469811| H, 7.3394347894,0.9326531862,1.563828 $6529 \mid$ H,9.9476201286,2.7619661075,2.7646723642| H,9.3900184084,3.640288172,1.3452930664| H,9 .4100822278,1.8651739754,1.3303427403| H,8.1343629616,3.9710886471,4.2349605838 | H,6.4349758 $675,3.9068469052,3.742839672 \mid$ H, 7.6025554302,4.857796006,2.8120597844| H,3.08688804768,2.4821 846101,3.5059687364| H,1.4839930091,1.9220047938,3 .0523616892 | H,2 .3039101578,3 .1377939487,2 $.0621382044 \mid$ | Version=AM64L-G03RevC.02 | State=1-A \HF=-1248.5016869 | RMSD=4.626e-09 | RMSF=3. 145e-06 | Dipole=0.4429376,0.0164821,-0.3691119 | PG=C01 [X(C30H5001) ] | | @

\section{$\mathrm{C}_{30} \mathrm{H}_{51} \mathrm{O}_{1}(+1)$ model for protosteryl C-13 cation}

1 | 1 | GINC-RN16 | FOpt | RB3LYP | 6-31G(d) | C30H5101(1+) | LINHAT | 14-Feb-2006 | 0 | | \# B3LYP/6-31G* FOPT GEOM=CHECK GUESS=READ IOP(1/8=5) | | protosteryl C-13 cation | | 1,1 | C, - 5.0608486544,-0.1477097253,0.3520736741 | C, - 5.2226462109, - 1.3693675972, - 0.54804269 | C, -4.4135143245,-2.606099027, -0.046994182 | C, -2.9202394696, -2.1364931907,0.1464169627 | C, $2.6786971853,-0.8682164937,1.0350257536|C,-3.5911652337,0.2657377651,0.4975649624| C,-1.962$ $3430477,-3.3083761884,0.4797312844|\mathrm{C},-0.5776164566,-2.8867567186,1.0222826221| \mathrm{C},-0.1215144$ $536,-1.5225774607,0.4810012646|\mathrm{C},-1.126106334,-0.4355323434,0.9977808094| \mathrm{C}, 1.3307946569,-1$ $.1509361779,1.0132108137$ | C, $1.6823573376,0.2456749987,0.6640847185$ | C, 0.6846785018,1.3288991 
28,0.6979303072 | C, -0.7766979402,0.9194450762,0.3594399828|C,2.5326340218, - 1.9807707529,0.4 $554700994 \mid$ C, 3.7240174443,-1.0100734929,0.4972712604 | C, 3.0897784979,0.3800195535,0.24750348 $95|0,-6.6214717761,-1.6165425712,-0.6340810531| \mathrm{C},-5.1015683842,-3.2319957432,1.1864997628$ | C, $-0.0237047375,-1.5611384587,-1.0715138594$ | C, $-4.4326823015,-3.6747630506,-1.1673403212$ | C, -3.0110529339,-1.1022215812,2.530104986 | C, 3.9725736848, 1.6126160005,0.6480536907 | C, 3.950 $1074393,1.8647800958,2.1642377356$ | C, 3.7244437958,2.9164315765,-0.1393114533 | C, 3.8240186627 ,2.7998224081,-1.6734676941 | C,3.6322837291,4.1265515069,-2.3600550875 | C,4 .5163705332,4.815 279463,-3.1002442092 | C,4.1385252959,6.1492548531,-3.6981633559|C, 5.9223851796,4.3678965866 ,$-3.415273442|\mathrm{C}, 1.4650862386,-1.1879728657,2.590323065|$ H, - 5.5174903826, - 0.350015976, 1.3270 768099 | H, - 5.6338602802,0.6783798383,-0.0829908908 | H, -4.8294342452, -1.1054178317, - 1.5470932 $608|\mathrm{H},-2.6586650283,-1.8029910341,-0.8655350852| \mathrm{H},-3.530535397,1.1433140698,1.1541812551 \mid$ H, -3.2304829092,0.5872767295,-0.4892169524| H, -1.822626702,-3.9078538366, - 0.424965948| H, - 2 . 4169183386,-3.9811196721,1.2142959354| H,0.1598447862,-3.6621150328,0.7782844528| H, -0.62050 80966,-2.8366484838,2.1154302495 | H, -0.8894604707, - - .3047284111,2.0574714026| H, 1.0209634443 ,2.1965748589,0.125238072| H,0.7126547064,1.6570512118,1.7570159825| H, - 1.4244968725,1.71686 42976,0.72923216| H, -0.8902029623,0.9065936884, - 0.7293272208| H, 2.3396099252, -2.2854508873, $0.5756701 \mid$ H, $2.6997832565,-2.8890200916,1.0393865003 \mid$ H, 4.4852221737, - 1.2405702565, - 0.252200 4856 | H, 4.2228548154,-1.0204986318,1.4723009702 | H,2.9199525521,0.4648350678,-0.8542567128 | H, - 6.7866842381, -2.2207301727, - 1.3731220638| H, -4.4641611302, - 3.9753373245, 1.6762989438| H, $6.0170862957,-3.7408131898,0.8736208815|\mathrm{H},-5.4029473536,-2.498950201,1.9363783273|$ H,0.6364 $911309,-0.7821865316,-1.4754507144 \mid$ H, - $0.9922433924,-1.4188256532,-1.5487242718 \mid$ H, 0.3662597

$659,-2.5213624688,-1.4220289253|\mathrm{H},-3.9800857473,-4.6170621056,-0.8435249996| \mathrm{H},-3.904593258$ 9,-3.3348682787, -2.0677100792| H, -5.4638952478, -3.9124826638, - 1.453372577| H, -2.74677646, - 0 . $2131572071,3.1165688399 \mid$ H, -2.4622687045,-1.9512921048,2.9513692324| H, -4.0698041574,-1.2887 998655,2.6999198027| H,4.9848977366,1.2782274967,0.3824200388| H,4.7052102379,2.6117188964,2 $.4278024748 \mid$ H,4.1747403359,0.9606972185,2.7409566898| H,2.9814277803,2 2.2552967733,2.4994992 $594 \mid$ H,4.4815312684,3.6358697297,0.1956211869| H,2.7619116186,3.3658185604,0.1405050807| H,3 $.0492179074,2.1048916393,-2.0438493926 \mid$ H,4.787139716,2.3499104678, - 1.9388111794| H,2.645662 7711,4.5701718413,-2.2144106298| H,4.8120320033,6.9417452714,-3.3451264894| H, 3.1129314723,6 $.4372622562,-3.4484470658|\mathrm{H}, 4.2307138997,6.1284419198,-4.7923764968| \mathrm{H}, 6.648589944,5.112120$ 2035,-3.0623719516| H,6.0649364801,4.2900484045,-4.5012852553| H,6.1906007734,3.4046037958,$2.9743661043|\mathrm{H}, 2.4539623279,-0.8607265757,2.9214512764| \mathrm{H}, 1.3427975135,-2.2305980431,2.8936$ 618591 | H,0.7171174273,-0.5801999969,3.0992898913 | | Version=AM64L-G03RevC.02 | Sta te=1-A | HF $=-1248.8419404 \mid$ RMSD=2.135e-09 | RMSF=3.892e-05 | Dipole=3.0309751,0.1021098,0.5440193 | PG=C01 $[\mathrm{X}(\mathrm{C} 30 \mathrm{H} 5101)]$ | | @

\section{$\mathrm{C}_{30} \mathrm{H}_{50} \mathrm{O}_{1}$ model for protosta-12,24-dien-3 $\beta$-ol}

1 | 1 | GINC-RN16 | F0pt | RB3LYP | 6-31G(d) | C30H5001 | LINHAT | 16-Feb-2006 | 0 | | \# B3LYP/6-31G* 
FOPT GEOM=CHECK IOP $(1 / 8=5)||$ protosta $-12,24-$ dien $-3 \beta-$ ol $\mid$

| 0,1 | C, - 5.4095899449,-1.8218654535,0.1282422922 | C, - 5.124066389,-3.190119338,-0.5004131857 | C, -3.8545815437, -3.9244873014,0.0510897556 | C, -2.703969961, -2.8601772271, - 0.0008433783 | C, $-2.9407800096,-1.5136628028,0.7635198723|\mathrm{C},-4.1977557161,-0.8696781139,0.1221178224| \mathrm{C},-1.2$ $595693173,-3.3312834274,0.2121990934|\mathrm{C},-0.247924811,-2.3487774269,-0.4532276634| \mathrm{C},-0.74351$ $61237,-0.8665089076,-0.6279476204|\mathrm{C},-1.6542816189,-0.5608664772,0.6145295798| \mathrm{C}, 0.503734387$ 6,0.1385280355,-0.7172950143 | C,0.9943381786,0.6716604318,0.6201483809 | C,0.4077863515,0.371 $1672863,1.7785060076 \mid \mathrm{C},-0.7648434123,-0.5571639992,1.8938811838$ | C, $0.2125490779,1.521030442$ 9,-1.3894790872 | C, 1.3847026958,2.4381825365,-0.9522231905 | C, 1.9046882016, 1.8836425086,0.41 80649022 | 0, - $6.3233633828,-3.9493702061,-0.3368147578 \mid$ C, - $4.1245204063,-4.6081344129,1.40982$ $74542|\mathrm{C},-3.5086940354,-5.0555459139,-0.9496487361| \mathrm{C},-3.257974131,-1.7008900322,2.267719397$ 6 | C,3.4476967196,1.6502039214,0.4910318377| C,4.2357149789,2.9579596691,0.7395620151 | C,4.1 878330243,4.0365989655,-0.3630060401|C, 5.0587506993,5.218997121,-0.0266245393 | C, 6.14965901 77,5.6710591866,-0.6645366708 | C,6.89744464989,6.8805772129,-0.1533772969| C,6.7373162341,5.0 608593877, - 1.9142106428 | C, 3.8273192302,0.6396918227, 1.5865113692 | C, 1.6637152927, - 0.5241776 $558,-1.5135497668|\mathrm{C},-1.4802277135,-0.6844126813,-1.9845664236| \mathrm{H},-5.7820261305,-1.978076692$ 2,1.1465833315| H, -6.242006835, - 1.3700846487, - - .4244237147| H, -4 .9412241168, -3.0250713723, - 1 $.5779709505|\mathrm{H},-2.746535796,-2.5871566096,-1.0566079681| \mathrm{H},-4.4615125515,0.0545048095,0.6547$ $563479|\mathrm{H},-3.977377801,-0.5753000435,-0.9110166737| \mathrm{H},-1.097209233,-4.3270279761,-0.21246005$ $37|\mathrm{H},-1.0489538305,-3.4293881078,1.2844066528| \mathrm{H}, 0.0315358312,-2.7437994727,-1.4369138328$ | H,0.6690259513,-2.3570121792,0.1443481375| H, -2.0403005557,0.4643583061,0.5134548672 | H,0.72 48383813,0.8840595525,2.6871046806| H, -0.4342302931, - - 1.5738508586,2.1432667663| H, -1.3536049 $907,-0.2281594216,2.7530155774 \mid$ H, - 0.7336179701,1.9232780725,-1.0084890452| H, 0.1308210979,1 $.4662896545,-2.4802907425|\mathrm{H}, 1.0563638639,3.4795534468,-0.8748744244| \mathrm{H}, 2.1812380638,2.42126$ $81267,-1.703041192|\mathrm{H}, 1.6717393338,2.5984229199,1.2185173254| \mathrm{H},-6.236354983,-4.762612239,-0$ $.8568622096|\mathrm{H},-3.1844875125,-4.8523278628,1.9176758499| \mathrm{H},-4.6691157493,-5.5459309919,1.257$ 3184032 | H, - 4.7325778715,-4.0092444189,2.0880257057| H, -2.7192147162,-5.7104013452, - 0.567391 $2426 \mid$ H, -3 .1816640931, -4.6612112859,-1.9199231817| H, -4.3803100058, - 5.6993670889, - 1.12787289 $65|\mathrm{H},-3.3303007127,-0.7262894529,2.762778034| \mathrm{H},-2.5063318693,-2.2916031002,2.7981684767 \mid \mathrm{H}$ $,-4.2164497034,-2.1896830705,2.4208129585|\mathrm{H}, 3.7853330976,1.2369943495,-0.4704561827| \mathrm{H}, 5.28$ $97359937,2.6928789017,0.8982334667 \mid$ H, 3.8935247489,3.3995266685, 1.6881735745 | H, 3. 1554564202 ,4.3882981221,-0.4885435414| H,4.4784456338,3.5902968477,-1.3209113012| H,4.7504825905,5.759 $161727,0.8713700362 \mid$ H, 7.9432220674,6.6321973933,0.0777170516| H,6.4394886106, 7.2923332949,0 .7516255485| H,6.929166453,7.6770583337,-0.9105537505| H,6.7532691345, 5.7932958731, -2.733654 $3473 \mid$ H, 6.1938029785,4 .1803376827, -2.2642993944| H, 7.7821659255,4 .7647735267, -1.745214265| H ,4.9125536235,0.4848180401,1.6084572336| H,3.3464150694, -0.3297690151,1.4303216963| H, 3.5246 189385,0.9991049169,2.5787968255| H,2.432819356,0.2061376393,-1.7731101028|H,1.3025322215, $0.950018636,-2.4560471744 \mid$ H,2.1540899559,-1.3229968018, - 0.9517110423 | H, - 1.9914878966,0.281 
$8099873,-2.0284930816|\mathrm{H},-2.2211935568,-1.4512214195,-2.203044506| \mathrm{H},-0.763422234,-0.7126365$ 782,-2.812243914 | | Vers ion=AM64L-G03RevC.02 | State=1-A | HF=-1248.4669198 | RMSD=5.489e-09 | R MSF=1.698e -06 | Dipole=0.449 4251,-0.1262551,-0.4303901| PG=C01 [X(C30H5001) ] | | @ 
Protosta-12,24-dien-3 $\beta$-ol ${ }^{1} \mathrm{H}$ NMR
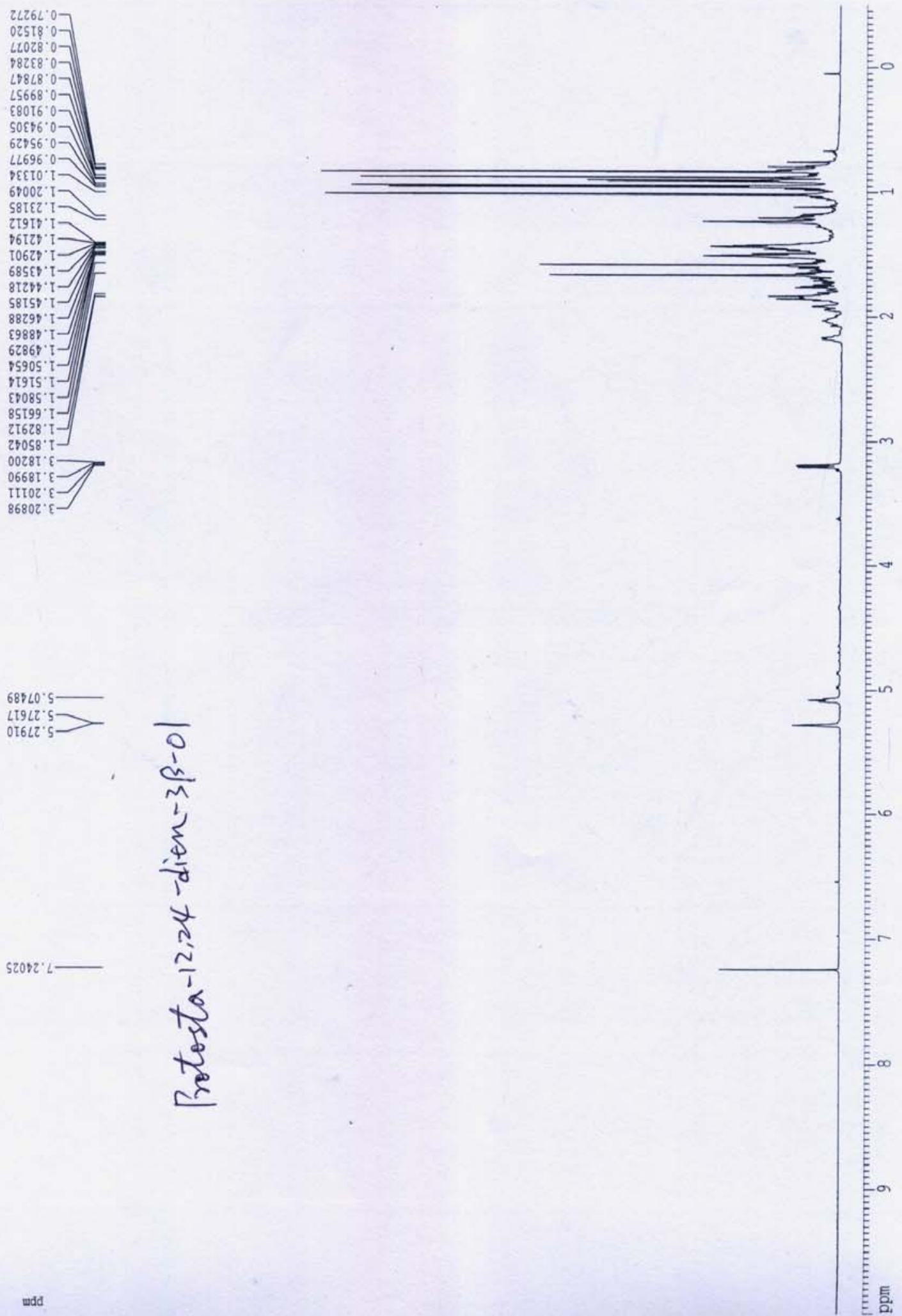
Protosta-12,24-dien-3 $\beta$-ol ${ }^{13} \mathrm{C}$ NMR

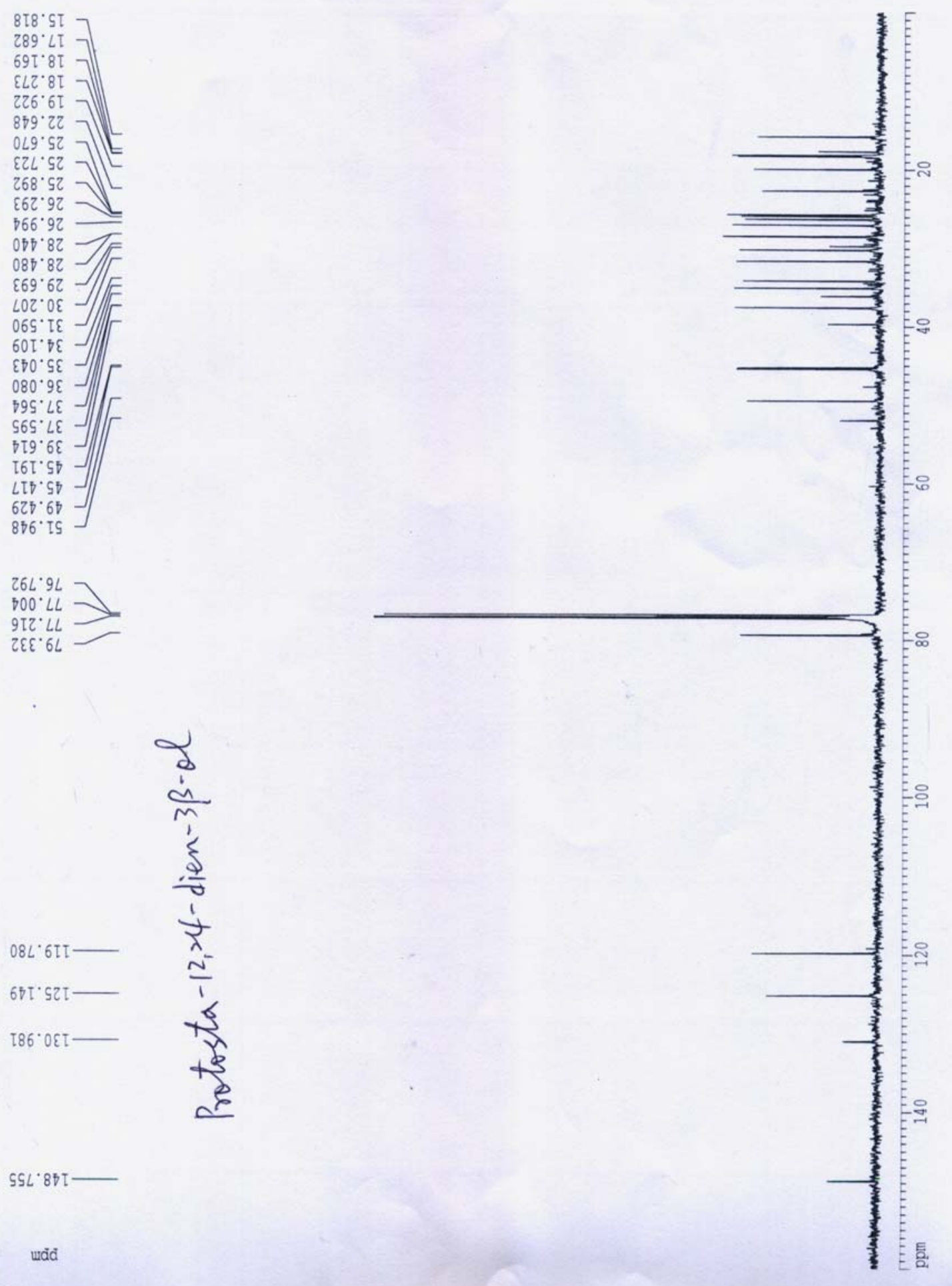


Protosta-12,24-dien-3 $\beta$-ol HSQC

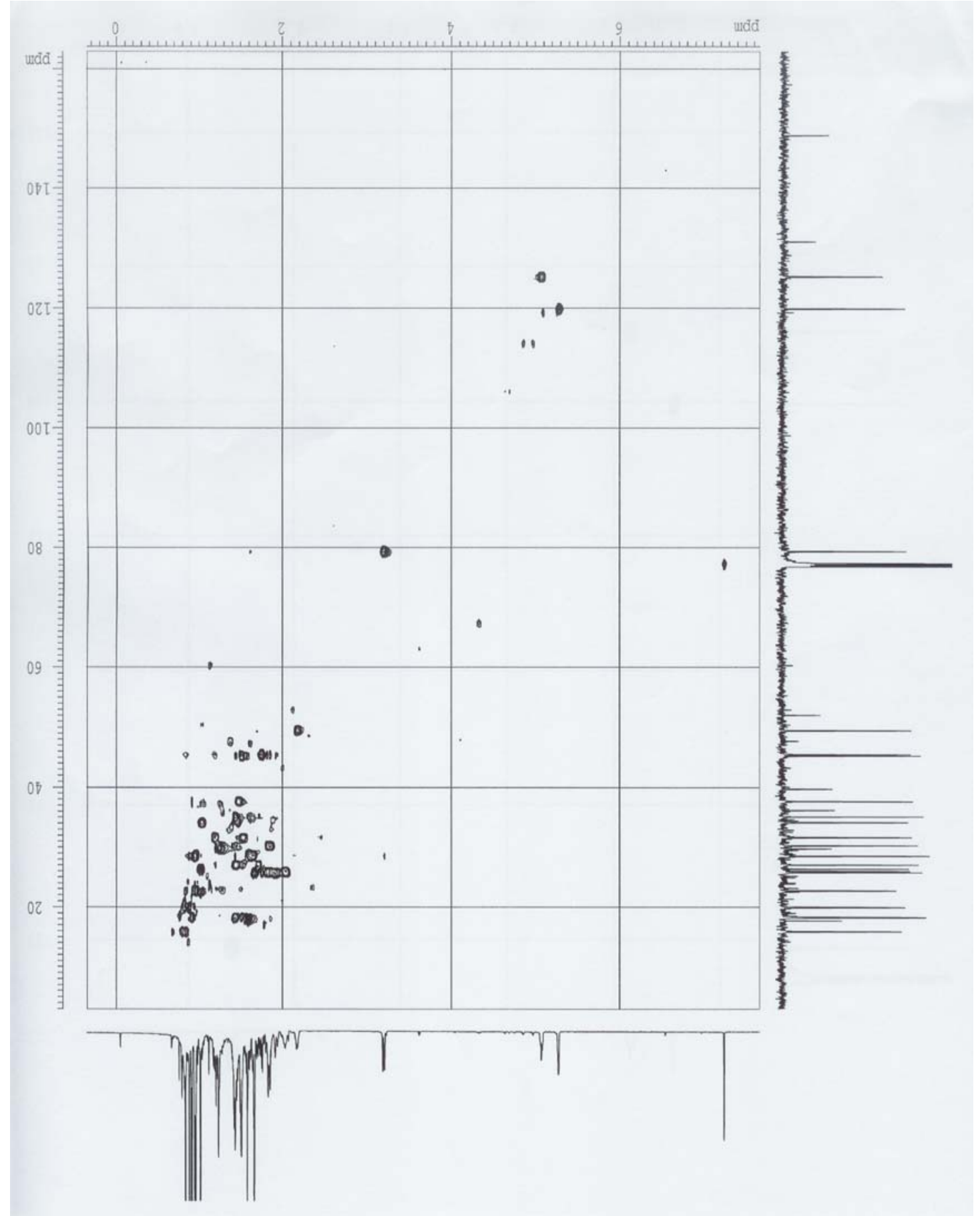


Protosta-12,24-dien-3 $\beta$-ol HMBC

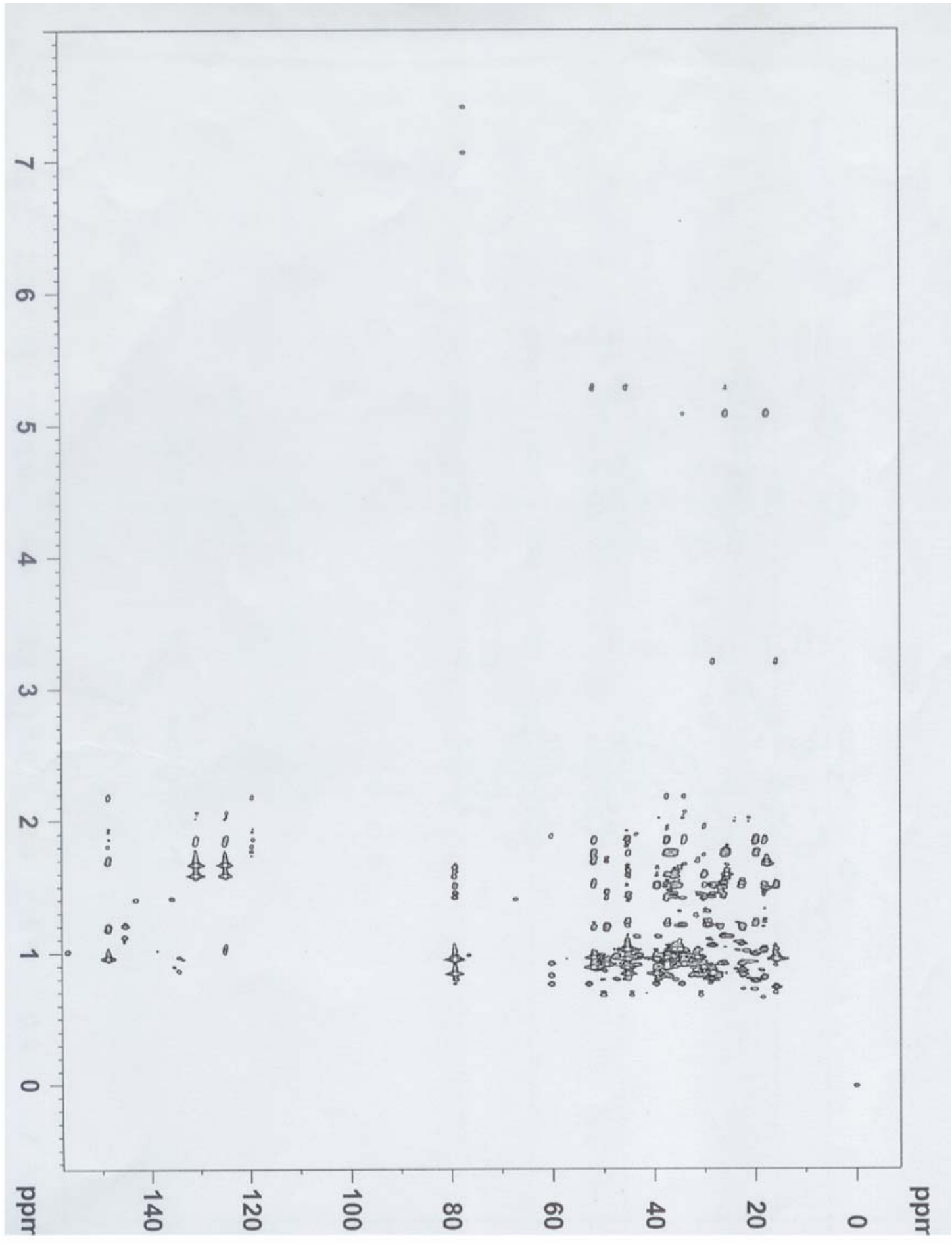


Protosta-12,24-dien-3 $\beta$-ol 2D-NOESY

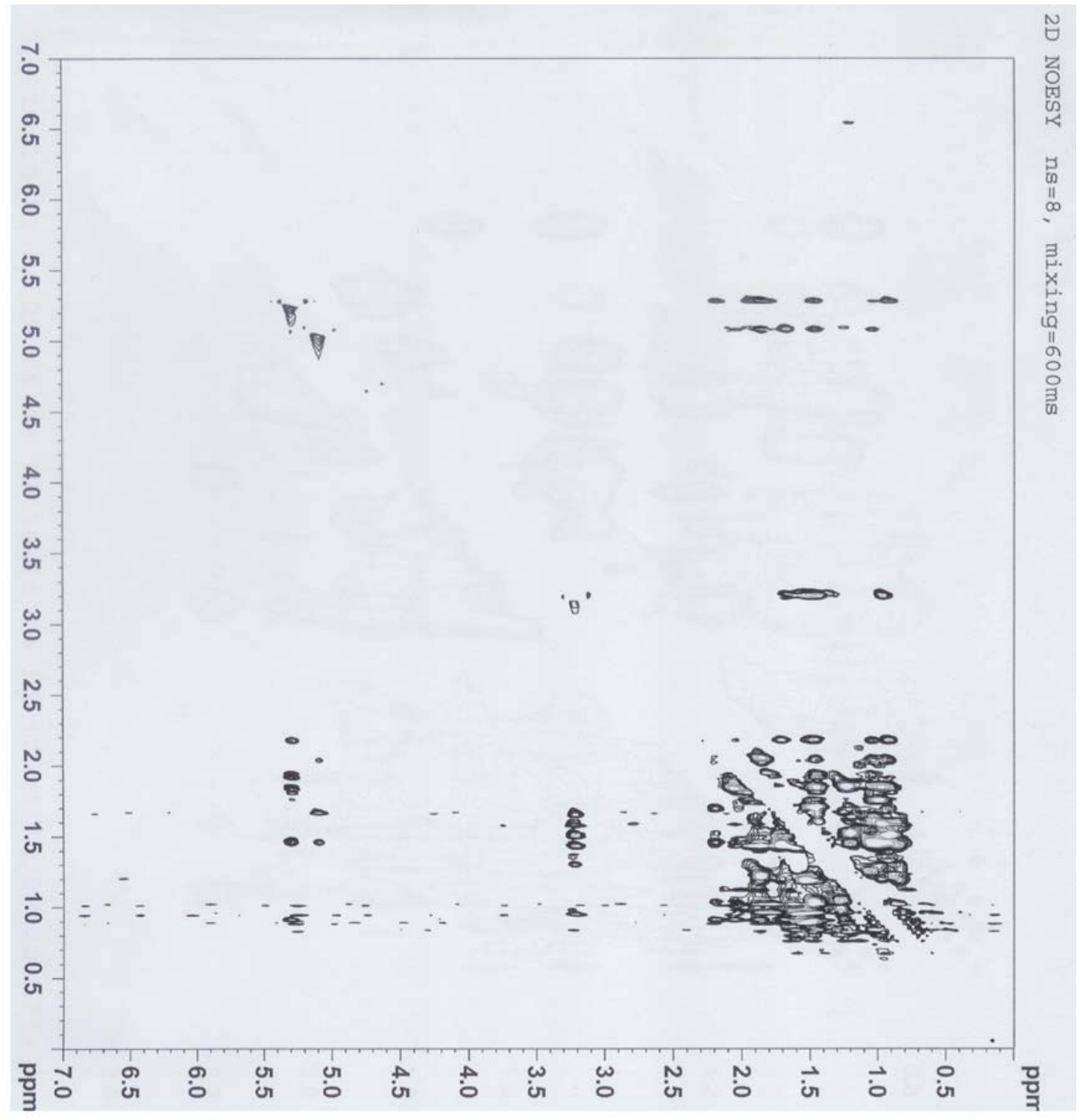




\section{Tryptophan 232 within \\ Oxidosqualene-Lanosterol Cyclase \\ from Saccharomyces cerevisiae \\ Influences Rearrangement and \\ Deprotonation but not Cyclization \\ Reactions}

Tung-Kung Wu ${ }^{\star a}$, Mei-Ting Yu ${ }^{\dagger a}$, Yuan-Ting Liu ${ }^{\dagger a}$, Cheng-Hsian

Chang $^{\dagger a}{ }^{\text {, Hsing-Ju Wang }}{ }^{\text {, }}$, and Eric Wei-Guang Diau ${ }^{b}$

a Department of Biological Science and Technology and ${ }^{\mathrm{b}}$ Department of Applied Chemistry

National Chiao Tung University

300, Hsin-Chu, Taiwan (Republic of China)

tkwmll@mail.nctu.edu.tw

Received Date (will be automatically inserted after manuscript is accepted)

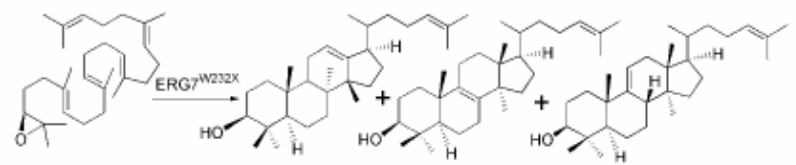

Oxidosqualene-lanosterol cyclases convert oxidosqualene to lanosterol in yeast and mammals. Site-saturated mutants' construction of Saccharomyces cerevisiae oxidosqualene-lanosterol cyclase, at Trp232 exchanges against proteinogenic amino acids, and product profiles were shown. All mutants, except Lys and Arg, produced protosta-

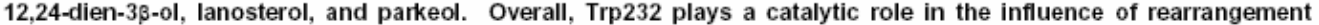
process and determination of deprotonation position, but not involves intervention in the cyclization steps.

The enzymatic conversion of acyclic oxidosqualene into diverse polycyclic sterols and triterpenoids, which is catalyzed by oxidosqualene cyclases, represents one of the most remarkable one-step biotransformation processes found in nature. ${ }^{1-3}$ Two biosynthetic routes, which have the following differences: folding of the substrate conformation, stabilization of the cationic intermediates and skeletal rearrangement, and deprotonation position, can be generally categorized and result in the production of diverse product profiles. This unique regio- and stereo-specific polyolefinic cyclization/rearrangement reaction mechanism, and the molecular interactions between cationic intermediates and functional groups of catalytic amino acid residues of the oxidosqualene cyclase enzymes, has attracted many biological chemists to study structure-function relationships of the enzyme. Genetic selection and truncated products isolation, from oxidosqualene cyclase mutants, have led to identification of critical amino acid residues, which are involved in either product specificity change from cycloartenol to lanosterol or determining cyclization/rearrangement cascade outcome. ${ }^{2,3}$ In addition, crystallographic analysis of both bacterial squalene-hopene cyclase (SHC) and human oxidosqualene-lanosterol cyclase (OSC) have provided a more in-depth analysis of structure-function relationships of (oxido)squalene cyclases., ${ }^{4,5 b}$ And yet, detailed molecular interactions between the substrate and enzyme for chair-boat 6-6 bicyclic B-ring and discrete 1,2hydride and methyl groups rearrangement, as well as deprotonation, are still far from being fully understood. 
Rearrangement steps during the oxidosqualene cyclasecatalyzed reaction were suggested to be driven by either $\pi$-electron density gradient, at different active site regions, or only minimal assistance from the enzyme. ${ }^{1 \mathrm{c}, 40,5}$ In one study, Corey used protostenediol benzoate as the substrate and non-enzymatically converted it to the corresponding dihydroparkeol benzoate, which suggested that minimal assistance is required of the enzyme during the rearrangement step. ${ }^{50}$ In the other study, Thoma et al proposed, from the human OSC X-ray structure, that the rearrangement reactions were driven by the $\pi$-electron density gradient in the different active site regions, and consequently terminated by the putative basic residue His 232 , when in the proximity of the termination site or the hydroxyl group of Tyr503, which is H-bonded to His 232 and located in a better position to accept protons from the C-9 of the lanosteryl cation. ${ }^{5 b}$ We previously performed site-directed mutagenesis experiments on Saccharomyces cerevisiae oxidosqualene-lanostero cyclase (ERG7) to elucidate putative amino acid residues that are involved in the cyclase-catalyzed deprotonation step. A putative base residue at the 510 position of ERG7, Tyr510 (corresponds to Tyr503 of human OSC), was mutated to alanine and the resulting $\mathrm{ERG} 7^{\mathrm{Y} 510 \mathrm{~A}}$ mutant was subjected to genetic complementation and product characterization $^{2 \mathrm{a}}$ The results obtained showed that the ERG $7^{\mathrm{Y} 510 \mathrm{~A}}$ mutant is viable and can generate multiple triterpene products, including an alternative deprotonation product parkeol. In addition, another putative base, His234 of ERG7 (corresponds to His232 of human OSC), was substituted with tyrosine and also resulted in a viable mutant and the isolation of multiple triterpenes, including incompletely cyclized achilleol A truncated rearranged protosta-12,24-dien-3 $\beta$-ol, lanosterol, and alternatively deprotonated product parkeol. $^{2 \mathrm{~b}}$ Conversely, lanosterol was isolated as the sole product from an $\mathrm{ERG} 7^{\mathrm{H} 234 \mathrm{Y} / \mathrm{Y} 510 \mathrm{~A}}$ double mutant. These findings support the hypothesis that the ERG7 His234:Tyr510 may function as the following: a catalytic base dyad in the control of final deprotonation, the plasticity of the dyad in response to other amino acid residues, and consequently in the change of product specificity. ${ }^{5 b}$ However, the possible involvement of other amino acid residues in cationic intermediates stabilization, during the rearrangement process or in the function as an alternative base for the final deprotonation step, could not be excluded.

To further elucidate possible involvement of other amino acid residues and their effect on the process of rearrangement and deprotonation, aromatic residues (with consensus of sequence among various oxidosqualene cyclases and spatially located between the C-20 and C8/C-9 position of lanosterol) were subjected for genetic selection and product characterization. This was performed with the assumption that the higher the $\pi$ electron density gradient in different active site regions in driving rearrangement process and their intrinsic reactivity to form cation- $\pi$ interactions to carbocationic intermediates. ${ }^{6}$ Among the various aromatic residues of
ERG7 under investigation, Trp232 is of particular interest. The performed sequence analysis provided the result that $\operatorname{Trp} 232$ is highly conserved in oxidosqualene-lanosterol cyclase, oxidosqualene-cycloartenol synthase, and $\beta$ amyrin synthase, but instead substituted by either leucine or glycine, within lupeol synthase and squalene-hopene cyclase, respectively. In addition, mutagenesis studies of ERG $7^{\text {w/232 }}$ performed by Corey and co-workers, which suggested that Trp232Ala, Trp232Gly, and Trp232Phe were functional, but could be chemically inactivated by the substrate analog, 20-oxa-2,3-oxidosqualene, which suggested a model for possible involvement of the residue in D-ring development. ${ }^{7}$ However, product isolation and characterization were not carried out with this mutant enzyme to investigate its catalytic reaction mechanism effect and product profiles. A detailed investigation of the structural model suggested contribution of higher $\pi$-electron density near the $\mathrm{C}$ - 12 position of lanosterol. Therefore, it is tempting to investigate effect of substituting Trp232 of ERG7 with Leu, Gly, and Ala, as well as other amino acids, for possible involvement of the residue during rearrangement steps. We report, within this context, results of sitesaturated mutation (W232X) and the product profile determination of each mutant. Identical product profile, which included truncated rearrangement product and lanosterol as well as parkeol, but with varying product proportions, was isolated from a strain that expressed ERG $7^{\text {w232X }}$ as its only oxidosqualene-cyclase. These results suggested a catalytic role for Trp232 in ERG7 that can influence both rearrangement process and deprotonation position, but is not involved in the cyclization steps

The ERG7 ${ }^{\mathrm{w} 232 \mathrm{X}}$ site-saturated mutants were constructed and expressed in a yeast HEM1 ERG7 double-knockout mutant TKW14, which is only viable when supplied with exogenous ergosterol or complemented with ERG7 activity. ${ }^{2 \mathrm{a}, 2 \mathrm{c}, 8}$ The TKW14[pERG7 $\left.{ }^{\text {W232X }}\right]_{\text {strain expressed ERG }}{ }^{\text {W232X }}$ as its only oxidosqualene cyclase. The genetic selection result demonstrated that TKW14[pERG $7^{\mathrm{w} 232 \mathrm{X}}$ ] site-saturated mutants allowed for ergosterol-independent growth, except for the ERG $7^{\mathrm{w} 232 \mathrm{~K}}$ and ERG $7^{\mathrm{W} 232 \mathrm{R}}$ mutations. These results indicated that the $\operatorname{Trp} 232$ mutations were not detrimental to the essential activity of ERG7, except for the Lys and Arg substitutions. Next, TKW14[pERG $\left.{ }^{\text {W232X }}\right]$ mutant strains were cultured individually, nonsaponifiable lipids (NSL) extracts were prepared, and products were analyzed by GC-MS, as described previously. ${ }^{2 b}$ The NSL extracts of TKW14[pERG7 $\left.{ }^{\mathrm{W} 232 \mathrm{x}}\right]$ mutants, that produced triterpenoid products with a molecular mass of $m / z=426$, were further acetylated and subjected to an $\mathrm{AgNO}_{3}$ impregnated silica gel column for chromatographic purification and structure characterization with NMR $\left({ }^{1} \mathrm{H}\right.$, ${ }^{13} \mathrm{C}$ NMR, DEPT, HSQC, HMBC, and 2D-NOE)

Product profiles of the ERG7 $7^{\mathrm{W} 232 \mathrm{X}}$ site-saturated mutations are summarized in Table 1. The $\mathrm{AgNO}_{3}-$ impregnated TLC and GC-MS results, from the NSL 
extracts, showed that no product with $m / z=426$ could be observed for $\mathrm{ERG} 7^{\mathrm{W} 232 \mathrm{~K}}$ and $\mathrm{ERG} 7^{\mathrm{W} 232 \mathrm{R}}$ mutations; consistent with ERG7 activity disruption. Conversely, three identical products, revealed on GC-MS with retention time and mass spectral characteristics consistent with that of protosta-12,24-dien-3 $\beta$-ol (POL), lanosterol (LA), and parkeol (PK), respectively, but with different product ratios, could be identified from the NSL extracts of other ERG7 ${ }^{\text {W232X }}$ mutants. Spectroscopic determination of each compound confirmed the above mentioned structures, showing spectra that proved to be consistent with those of authentic compounds. ${ }^{2 \mathrm{~b}}$ Of all the $E R G 7^{\mathrm{W}_{232 \mathrm{X}}}$ mutants studied, the ERG7 ${ }^{\mathrm{W} 232 \mathrm{Y}}$ mutant biosynthesized LA most accurately, whereas the W232L mutation favored PK and POL formation more than LA production. Interestingly, no monocyclic achilleol $\mathrm{A}$ or camelliol C was observed in the GC-MS analyses from any of the mutants.

Table 1. The product profiles of S. cerevisiae TKW14 expressing the ERG ${ }^{\text {W232X }}$ site-saturated mutants.

\begin{tabular}{|c|c|c|c|}
\hline $\begin{array}{l}\text { amino acid } \\
\text { sutsatifutien }\end{array}$ & 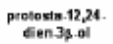 & Ianesteral & parkeal \\
\hline $\begin{array}{l}\text { nestive } \\
\text { W2726 }\end{array}$ & 89 & $\begin{array}{l}160 \\
74.4\end{array}$ & in \\
\hline weran & 18. 1 & 47.1 & 34.8 \\
\hline weว32 & 19.3 & 34.0 & 46.7 \\
\hline wo321 & 23.5 & 330 & 435 \\
\hline w232L & 16.5 & 28.8 & 583 \\
\hline W2320 & 19 & 9.8 & 13 \\
\hline W232E & 16.0 & 492 & 36.8 \\
\hline wezes & 90.7 & s9. & 30.3 \\
\hline พ232T & 10.3 & 61.3 & 24.0 \\
\hline พอะ324 & 10.7 & 595 & 29.8 \\
\hline wom2a & 24.6 & 32.1 & 433 \\
\hline & \multicolumn{3}{|c|}{ 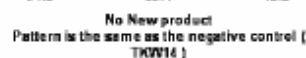 } \\
\hline werc & พิ8.0 & 31.8 & 50.2 \\
\hline พЕะзан & 27.8 & 35.0 & 372 \\
\hline wอะ32P & 19.8 & 485 & 32.1 \\
\hline n2032F & 4.4 & $n 29$ & 127 \\
\hline NDZ32Y & 42 & 9.2 & 1.5 \\
\hline W232M & 10.9 & 40.5 & 48.5 \\
\hline
\end{tabular}

How nature evolved the $E R G 7$ gene in the eukaryote to form lanosterol, as the sole product, remains unknown. On the other hand, plant oxidosqualene-cyclases utilized the same substrate, but generated diverse product arrays, with similar core structures. In ERG $7^{\text {w232x }}$ mutants, oxidosqualene is cationic cyclized to a tetracyclic protosteroyl $\mathrm{C}-20$ cation, without disruption at the monocyclic $\mathrm{C}-10$ cation position. Then, a backbone rearrangement of $\mathrm{H}-17 \alpha \rightarrow 20 \alpha, \mathrm{H}-13 \alpha \rightarrow 17 \alpha, 1,2$-hydride shifts to generate the $\mathrm{C}-13$ cation, with elimination of $\mathrm{H}$ 12 , yielding protosta-12,24-dien-3 $\beta$-ol with the $\triangle^{12}$ double bond. Skeletal rearrangement of two methyl groups, originally derived from $\mathrm{C}-14 \beta$ and $\mathrm{C}-8 \alpha$ positions, and one hydride shift from $\mathrm{H}-9 \beta$ to $\mathrm{H}-8 \beta$, will generate the lanosteryl C-8/C-9 cation, which undergoes deprotonation at $\mathrm{C}-8$ and $\mathrm{C}-11$ to form lanosterol and parkeol, respectively (Scheme I)

With only a limited understanding of variability in metabolic flux ratios of POL, LA, and PK within the in vivo system, the product profiles reported herein provide some qualitative insights into the ERG7-catalyzed cyclization/rearrangement mechanism. We provided a plausible explanation as to how the substitution of Trp232, with other amino acid residues, impacts the difference in stabilization of rearrangement process and regulates deprotonation postions by use of structure models derived from human OSC crystal structure and complexed with lanosterol and protosteryl C-13 cation structure, respectively. ${ }^{2 \mathrm{a}, 2 \mathrm{~b}, 40,9}$ The structure of protosteryl $\mathrm{C}-13$ cation was constructed and optimized at the B3LYP/6-31G* level of theory using Gaussian 03 software, reasoning the difference in spatial loactions of the methyl groups at $\mathrm{C}-8$ and $\mathrm{C}-14$ positions, between POL and C-8/C-9 carbocation products, LA and PK. Similar structure integrity was observed between lanosterol-complexed and protosteryl C-13 cationcomplexed homology models, in both the distribution of secondary structure and 3-D profile (Figure 1).

Scheme 1. Product profile produced by $S$. cerevisiae TKW14 expressing the ERG7 ${ }^{\text {W232X }}$ sitesaturated mutations.

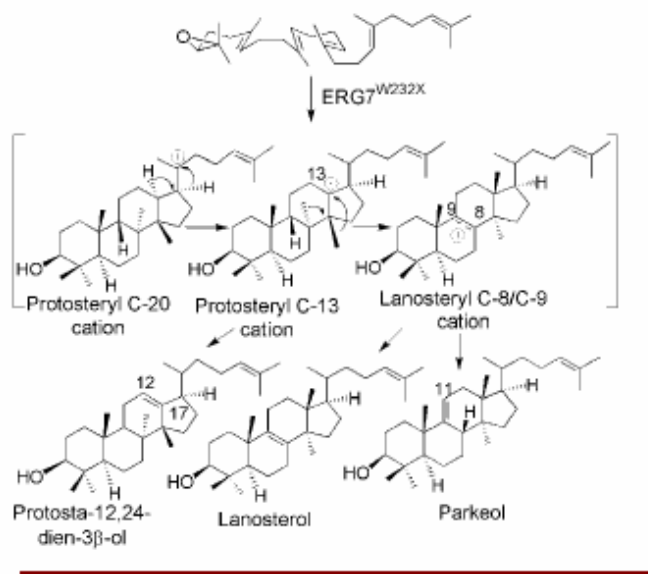

As previously established, His 234 and the Tyr510 residues of $S$. cerevisiae ERG7 are strictly spatial, as conserved with corresponding residues, within the human OSC, and that there is consistence between the mutant homology model and the mutant product profile distribution. $^{2}$ Careful examination of the homology model suggested that Trp232 is positioned at the top half of the side wall of the active site cavity and proximal to $\mathrm{C}-11, \mathrm{C}-12$, and $\mathrm{C}-13$ orientations of the products. In addition, the Trp 232 is also spatially close to His 234 and 
Tyr510, which occupy part of the ceiling of the active site and are H-bonded to each other. Homology simulation of the Trp232 position with other residues showed that changes at this position reduce the $\pi$-electron density nearby and affect the interactions of active site residues, especially the His234:Tyr510 catalytic base dyad $\mathrm{H}$ bonding network, as well as shift the relative distance between His 234 and $\mathrm{C}-11 / \mathrm{C}-12$ position of LA. For example, substitution of $\operatorname{Trp} 232$ with non-polar hydrophobic side chains such as Gly, Ala, Val and Ile enlarged the cavity of the active site. This, in turn, impaired the His 234:Tyr510 catalytic base dyad Hbonding network, and slightly pulled His 234 away from its original position and toward the $\mathrm{C}-11$ and $\mathrm{C}-12$ position to form $\mathrm{PK}$ and $\mathrm{POL}$, respectively. Consistent with this observation is the production of more $\mathrm{PK}+\mathrm{POL}$ than LA, as the side chain length was increased from $\mathrm{H}$ to the isobutyl group (Table 1). Alternatively, the change from Trp232, to Tyr or Phe, retained the His234:Tyr510 $\mathrm{H}$-bonding network and only slightly reduced active site steric hindrance and the $\pi$-electron density. This minor reduction of the ligand-enzyme interaction resulted in LA production as the major product, but with production of minor amounts of PK and POL.
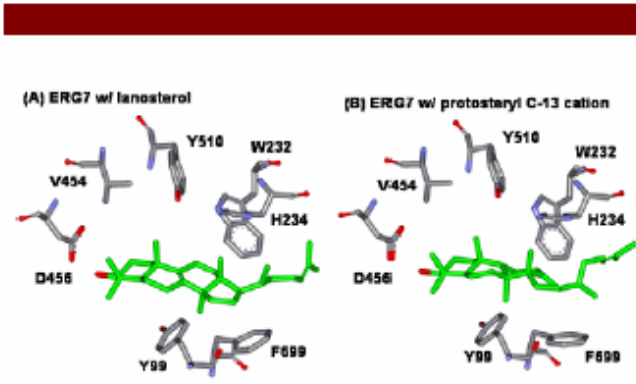

Figure 1. ERG7 residues interacting with $\mathrm{C}-11 / \mathrm{C}-12$ of lanosterol and prtosteryl $\mathrm{C}-13$ cation, respectively, in the homology model structures. A) ERG7 complexed with lanosterol and B) ERG7 complexed with prtosteryl C-13 cation.

It has been suggested that a higher $\pi$-electron density, near the $\mathrm{C}-8 / \mathrm{C}-9$ positions of $\mathrm{LA}$ with seven aromatic residues, might be responsible for the equilibrium shift toward the C-8/C-9 cation and, subsequently, for the lanosterol formation. ${ }^{5 \mathrm{~b}}$ In ERG $7^{\mathrm{w} 232 \mathrm{X}}$ mutants, partial deprotonation, for POL formation, occurred prior to the shift of two methyl groups (Me-14 $\beta \rightarrow \mathrm{Me}-13 \beta$ and Me$8 \alpha \rightarrow \mathrm{Me}-14 \alpha$ ) for generating lanosteryl C-8/C-9 cation and subsequent formation of LA and PK. Quantum mechanical calculation showed that formation of LA and PK are thermodynamically favored by about 21.82 and $21.36 \mathrm{kcal} / \mathrm{mol}$, respectively, relative to $\mathrm{POL}$ (see the Supporting Information). ${ }^{9}$ In addition, direct deprotonation of protosteryl C-13 cation to form protosta- 12,24-dien-3 $\beta$-ol were thermodynamically unfavorable. Perhaps the substitution of Trp232, with other amino acid residues, resulted in the loss of cation- $\pi$ interactions, which would destabilize the carbocation and facilitate deprotonation. Alternatively, the mutations at Trp232 would lead to stabilization or destabilization of various carbocation intermediates relative to the wild-type enzyme, which subsequently affects the relative rates of deprotonation and rearrangement. Further studies should focus on elucidation of precise molecular interactions on relative rates of deprotonation and rearrangement.

These performed experiments demonstrated plasticity of the ERG7 mutant enzyme and that mutations, at Trp232 position, change active site structure. This led to stabilization or destabilization of various carbocation intermediates, reative to the wild-type enzyme, and impaired catalytic fidelity of the enzyme, resulting in generation of multiple triterpene regioisomers. However, exact steric or electrostatic contributions of the mutations on the trajectories of product proportions, and rates for different products formation, remain unclear, awaiting creation of high-resolution crystal structures of mutated proteins, as well as kinetic analysis, which is currently under investigation.

Acknowledgment We thank the National Chiao Tung University and the National Science Council of the Republic of China for financial support of this research under Contract No. NSC-93-2113-M-009-012. helpful advice. We thank Prof. Jinn-Moon Yang for providing essential guidance on bioin formatic calculations.

Supporting Information Available Information supporting this article is available on the WWW under httn://wwwondettarg or is available from the author

(1) (a) Abe, I.; Rohmer, M.; Prestwich, G. D. Chem. Rev. 1993, 93, 2189-2206. (b) Xu, R.; Fazio, G. C.; Matsuda, S. P. T. Phytochemistry 2004, 65, 261-291. (c) Wendt, K. U.; Schulz, G. E.; Corey, E. J.; Liu, D. R. Angew. Chem. Int. Ed. 2000, 39, 2812-2833.

(2) (a) Wu, T. K.; Chang, C. H. ChemBioChem 2004, 5, 1712-1715. b) Wu, T. K.; Liu, Y. T.; Chang, C. H. ChemBioChem 2005, 6, 1177 1181. (c) Wu, T. K.; Griffin, J. H. Biochemistry 2002, 41, 8238-8244. (d) Wu, T. K.: Huang, C. Y.: Ko, C. Y.; Chang, C. H.: Chen, Y. J.; Liao, H. K. Arch. Biochem. Biophys. 2004, 421, 42-53. (e) Wu, T. K.

(3) (a) Hart, E. A.; Hua, L.; Darr, L. B.; Wilson, W. K.; Pang, J.; Matsuda, S. P. T. J. Am. Chem. Soc. 1999, 121, 9887-9888. (b) Joubert, B. M.; Hua, L.; Matsuda, S. P. T. Org. Lett. 2000, 2, 339-341. (c) Matsuda, S. P. T.; Darr, L. B.; Hart, E. A.; Herrera, J. B. R.; McCann, K. E.; Meyer, M. M.; Pang, J.; Schepmann, H. G.; Wilson, W. K. Org. Lett. 2000, 2, 2261-2263. (d) Herrera, J. B. R.; Wilson, W. K.; Matsuda, S. P. T. J. Am. Chem. Soc. 2000, 122, 6765-6766. (e) Mejer, M. M.: Xu, R.; Matsuda, S. P. T. Org. Lett. 2002, 4, 1395-1398. (f) Lodeiro, S.; SchulzGasch, T.: Matsuda, S. P. T. J. Am. Chem. Soc. 2005, 127, 14132-14133. (4) (a) Wendt, K. U.; Poralla, K.; Schulz, G. E. Science 1997, 277 , 1811-1815. (b) Wendt, K. U.; Lenhart, A.; Schulz, G. E. J. Mol Biol. 1999, 286, 175-187. (c) Wendt, K. U. Angew. Chem. Int. Ed. 2005, 44, $3966-3971$ (5) (a) Corey, E. J.; Virgil, S. C. J. Am. Chem. Soc. 1990, 112, $6429-$
6431. (b) Thoma, R.; Schulz-Gasch, T.; D'Arcy, B.; Benz, J.; Dehmlow, H.; Hennig, M.; Stihle, M.; Ruf, A. Nature 2004, 432, 118-122.

(6) (a) Buntel, C. J.; Griffin, J. H. J. Am. Chem. Soc. 1992, 114, 9711 9713. (b) Dougherty, D. A. Science 1996, 271, 163-168. (c) Lesburg, C. A.; Caruthers, J. M.; Paschall, C. M.; Christianson, D. W. Curr. Opin. Struct. Biol. 1998, 8, 695-703.

(7) Corey, E. J.; Cheng, H.; Baker, C. H.; Matsuda, S. P. T.; Li, D.; Song, X. J. Am. Chem. Soc. 1997, 119, 1289-1296

(8) Shi, Z; Buntel, C. J.; Griffin, J. H. Proc. Natl. Acad. Sci.U.S.A $1994,91,7370-7374$.

(9) Gaussian 03, Revision C.02, Frisch, M. J. et al Gaussian, Inc., Wallingford CT, 2004. 